\title{
Switchable Multimode Microlaser based on AIE
}

\section{Microsphere}

Fengyan Song, Chunhuan Zhang, Haiyun Dong, Yuqin Fan, Ming-Yu Wu, Guogang Shan,

Puxiang Lai, Hui Gao*, Yong Sheng Zhao*, Sijie Chen*

Dr. F. Song, Prof. H. Gao

School of Aeronautic Science and Engineering, Beihang University, Beijing 100191, China

E-mail: $\underline{\text { h.gao@ buaa.edu.cn }}$

Dr. C. Zhang, Dr. H. Dong, Dr. Y. Fan, Prof. Y. S. Zhao

Key Laboratory of Photochemistry, Institute of Chemistry, Chinese Academy of Sciences,

Beijing 100190, China

E-mail: yszhao@iccas.ac.cn

Prof. H. Gao, Dr. M-Y, Wu, Dr. S, Chen

Ming Wai Lau Centre for Reparative Medicine, Karolinska Institutet, Hong Kong, China

E-mail: sijie.chen@ki.se

Dr. G. Shan

Institute of Functional Material Chemistry, Faculty of Chemistry, Northeast Normal University, Renmin Road 5268, Changchun 130024, P.R. China.

Dr. P. Lai 
Department of Biomedical Engineering, The Hong Kong Polytechnic University, Hong Kong, China

KEYWORDS: Aggregation-induced emission, intramolecular charge transfer, organic microlaser, switchable laser, stimulus response materials.

ABSTRACT: Switchable multimode microlasers are of great significance to the development of photonic devices with high integration levels. Herein, we demonstrate an acid/alkaline gas responsive multimode AIEgen@starch microsphere-based microlaser. The aggregation-induced emission (AIE) active fluorescent dye ASCPI is used as the gain medium in this study. ASCPI was weakly emissive in water but became highly emissive when introduced to the starch microsphere as a guest molecule. The resultant ASCPI@starch microsphere worked well as a typical whispering-gallery-mode microlaser. The laser mode wavelengths were size dependent. Due to the sensitivity of ASCPI to $\mathrm{pH}$, the output wavelength of the microlaser could be switched to shorter wavelength by acetic acid vapor treatment or longer wavelength by $\mathrm{NH}_{3}$ vapor treatment. This work will provide a useful enlightenment for the rational design of effective switchable lasers using AIE materials with a simple preparation procedure.

\section{INTRODUCTION}

Miniaturized lasers have gained an increasing interest owing to their applications in chemical and biological sensing, ${ }^{1-3}$ laser display, ${ }^{4-6}$ imaging $^{7}$, and on-chip optical interconnects ${ }^{8-11}$. Wavelength-tunable lasers with capability of delivering intense coherent light signals across broad spectral range represent particularly interesting opportunities for highly integrated photonic devices. Compared to inorganic material based lasers, organic materials with tailorable excitedstate processes and facile fabrication offer an alternative to realize wavelength-switchable 
microlasers. ${ }^{12-15}$ To date, wavelength tunable lasers based on organic materials can be realized by modulating the energy level of excited state ${ }^{16-20}$ or mode variation of the optical resonator ${ }^{13,21-23}$. The excited-state energy level of gain materials can be tuned by external stimuli including humidity ${ }^{24}$, temperature ${ }^{19,25}$, light $^{26}$, vapor $^{16-17}$, solvent ${ }^{27}$, etc.. However, reports on gas responsive micro-/nanolasers are limited. ${ }^{16-17,27}$

Aggregation-induced emission luminogens (AIEgens) are new type of luminescent materials which are non-emissive when molecularly dissolved, but induced to luminesce by aggregate formation. ${ }^{28-32}$ AIEgens used as optical gain materials of microlasers can take advantages of the inherent AIE characteristics to improve lasing performance including dramatic threshold reduction and favorable lasing stability. ${ }^{33}$ In addition, these AIEgen-based miniaturized lasers can improve their laser performance with much higher doping concentration. ${ }^{33}$ However, there are only few reports of AIEgen-based miniaturized lasers. ${ }^{33-34}$ The environmental-responsive emission behavior of AIEgen-based miniaturized lasers was more elusive in the literature. Excited-state intramolecular charge transfer (ICT) ${ }^{35-38}$ effect endows the AIEgens with high sensitivity to the microenvironment. Therefore, AIEgens with ICT effect might be ideal candidates to fabricate robust wavelength-switchable microlasers.

Herein, we report a switchable multimode microlaser based on the ASCPI@ starch microsphere. The cationic AIEgen, ASCPI, was originally weakly emissive in water solution due to its AIE and ICT properties. When mixed with starch granules, ASCPI could readily bind to the starch. The host-guest interaction restricted the intramolecular motion of the AIE molecules and strong fluorescence was triggered with blue-shifted emission color. The acid gas could protonate ASCPI, which would consequently weaken the electron density of the donor system and therefore led to blue shift of gain region. In contrast, alkaline gas $\left(\mathrm{NH}_{3}\right)$ provided a more polar environment for 
these ICT chromophores and thus led to a red-shifted gain behavior. As a result, we discover a method of tuning lasing wavelength in the starch microparticles based on the acid/base-responsive AIEgen. These results offer a novel understanding on the stimulated emission of AIEgens and provide a useful enlightenment for the rational design of miniaturized lasers with desired performance.

\section{EXPERIMENTAL SECTION}

2.1. AIEgen preparation. AIEgen (ASCPI) was synthesized according to the previous literature. ${ }^{39}$ 2.2. Extraction Process of Potato Starch Granules. Potato starch granules were extracted from a fresh potato purchased at a local market. The potato was sliced into flakes, soaked in pure water, and vigorously shaken for 30-60 mins. Potato starch granules were collected by centrifuging the solution (4000 rpm for $5 \mathrm{~min}$ ), followed by further washing and purification through three times centrifugation with pure water. Finally, the as-prepared fresh potato starch granules were collected and dried in oven at $40{ }^{\circ} \mathrm{C}$.

2.3. Preparation of ASCPI@starch. $0.4 \mathrm{mg}$ starch granules were immersed in $1 \mathrm{~mL}$ of pure water and sonicated for $5 \mathrm{~s}$. Then $10 \mu \mathrm{L}$ of ASCPI $\left(10^{-3} \mathrm{M}\right.$ in DMSO) was dropped into the starch/ $\mathrm{H}_{2} \mathrm{O}$ solution, shaken by hand and left at ambient temperature for $24 \mathrm{~h}$. The the mixture was centrifagued and washed with pure water for several times until no characteristic emission was observed in the supernatant upon excitation, and then dried in oven at $40{ }^{\circ} \mathrm{C}$.

2.4. Measurements. The absorption and fluorescence spectra were measured with PerkinElmer Lambda-950 spectrophotometer and Edinburgh FLS-1000 instruments, respectively. The timeresolved photoluminescence (TRPL) was measured with Edinburgh FLS-1000 spectrofluorometer system equipped with EPLED-360. The absolute luminescence quantum yield were measured on 
a Edinburgh FLS 980 fluorescence spectrophotometer equipped with an integrating sphere $(0.1$ $\mathrm{nm}$ step size, 0.3 second integration time, 5 repeats).The morphology of the microsphere was examined with scanning electron microscopy (SEM, FEI Nova NanoSEM450). X-ray diffraction measurements were conducted on a D/max-2550 PC X-ray diffractometer with $\mathrm{Cu}$ Ka radiation. Bright-field optical images and fluorescence microscopy images were taken from an inverted fluorescence microscope (Nikon Ti-U), by exciting the samples with a mercury lamp. Lasing measurements were performed on a home-built far-field microphotoluminescence system. The microsphere was locally excited with a focused $400 \mathrm{~nm}$ femtosecond laser (fs-laser), which was generated from the second harmonic of the fundamental output of a regenerative amplifier (Solstice, Spectra-Physics, $800 \mathrm{~nm}, 100 \mathrm{fs}, 1 \mathrm{kHz}$ ).

\section{RESULTS AND DISSCUSSIONS}

A typical AIEgen sensitive to $\mathrm{pH}$ was chosen as a model molecule to demonstrate the concept. Figure 1a shows the chemical structure of the AIEgen, 4-(4-(1-cyano-2-(4(dimethylamino)phenyl)vinyl)phenyl)-1-methylpyridin-1-ium iodide (ASCPI). The ASCPI was synthesized according to the previous method. ${ }^{39}$ As illustrated in Figure 1b, a typical AIE property was observed. Its diluted solution in DMSO exhibited a weak photoluminescence (PL) with an emission peak at $674 \mathrm{~nm}$. Upon the addition of a non-polar solvent of 1,4-dioxane as the poor solvent for ASCPI into DMSO solution with a fraction of 90 vol \%, bright red PL with a maximum emission at $650 \mathrm{~nm}$ was observed. In addition, the $\alpha_{\mathrm{AIE}}$ value of ASCPI (Figure 1c) was calculated to be 9.3 when $f_{\mathrm{d}}(1,4$-dioxane fraction $)=90 \%$, suggesting a typical AIE feature for this molecule. Moreover, ASCPI demonstrated a large Stokes shifts $(\Delta v=192 \mathrm{~nm})$, which can be attributed to an excited-state ICT between the electron donor and the electron acceptor. The ICT effect was also 
confirmed by the experiment of solvatochromic effects ${ }^{36-37}$ (Figures S1, Supporting Information). The ASCPI presented varied emission spectra in different solvents with wavelength changing from $666 \mathrm{~nm}$ to $688 \mathrm{~nm}$, indicating a typical charge transfer character in the excited state (Figure S1b, Supporting Information).

a<smiles>CN(C)c1ccc(/C=C(\C#N)c2ccc(-c3cc[n+](C)cc3)cc2)cc1</smiles>

d

ASCPI

$\because \cdots$
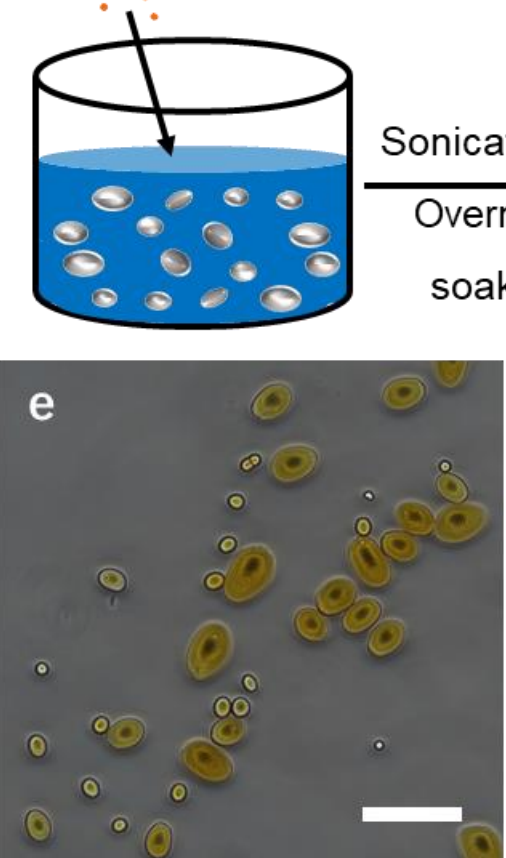

b

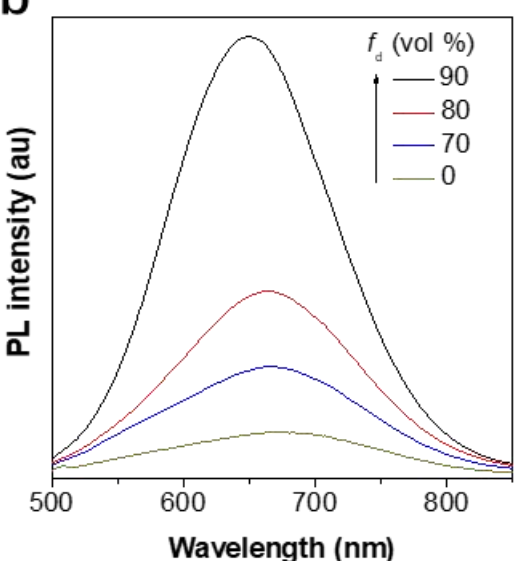

C

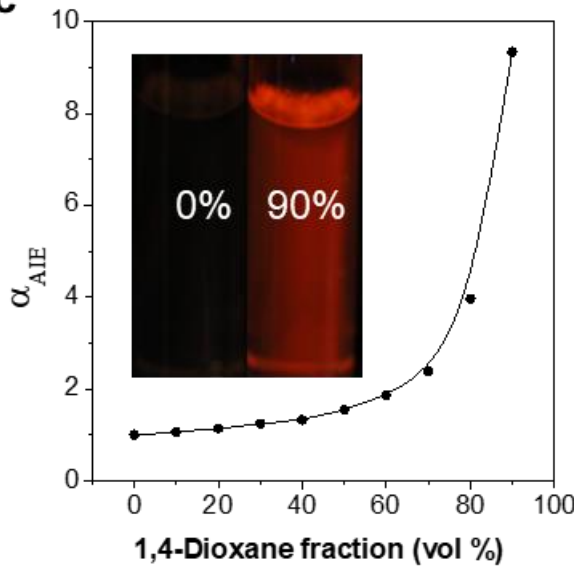

ASCPI@starch Microparticles
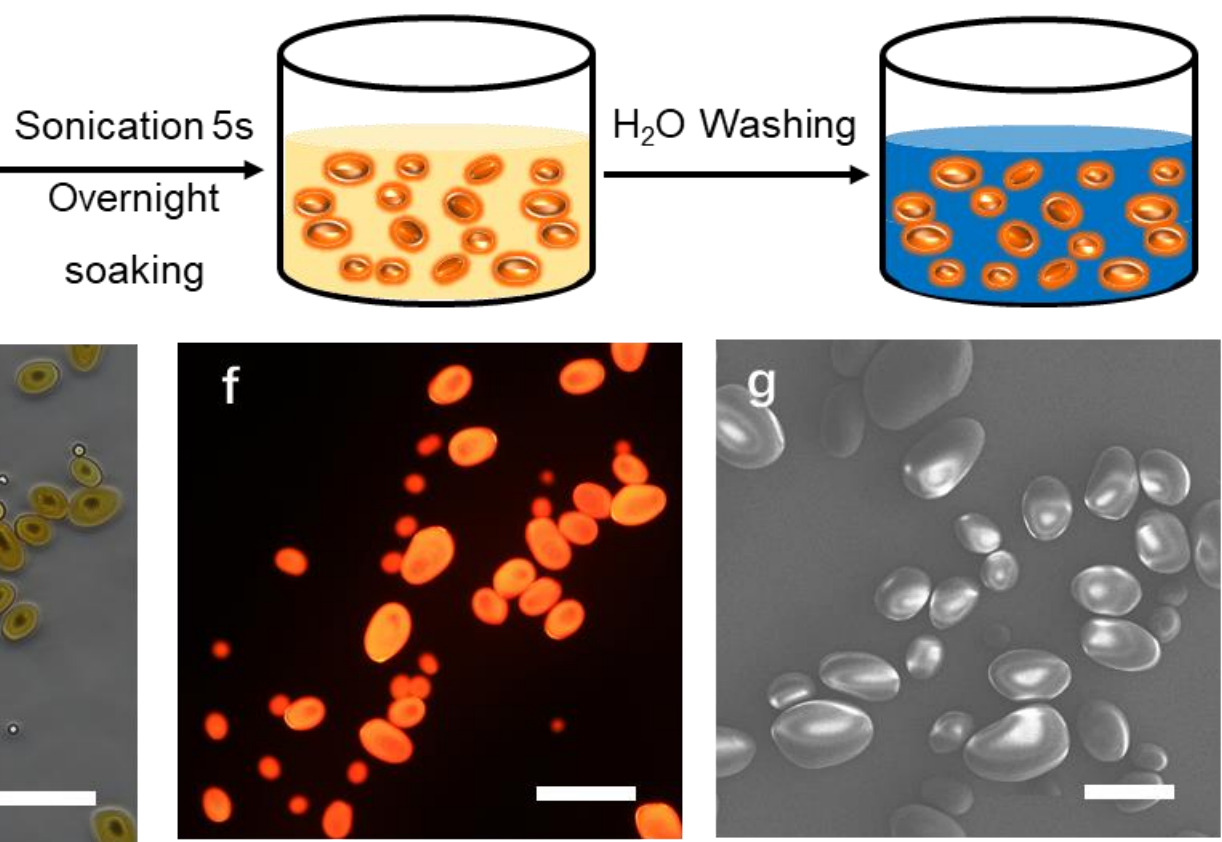

Figure 1. a) Molecular structure of ASCPI. b) PL spectra of ASCPI in DMSO/1,4-dioxane mixtures with different 1,4-dioxane fractions $\left(f_{\mathrm{d}}=0,70 \%, 80 \%, 90 \%\right)$. c) Plot of relative emission 
peak intensity of ASCPI ( $\left.\mathrm{a}_{\mathrm{AIE}}\right)$ versus $f_{\mathrm{d}}$ of DMSO/1,4-dioxane mixtures, where $\mathrm{a}_{\mathrm{AIE}}=I / I_{0}, I=$ emission intensity and $I_{0}=$ emission intensity in DMSO solution. Inset: fluorescence image of ASCPI in DMSO/1,4-dioxane mixtures with different $f_{\mathrm{d}}$ state taken under $365 \mathrm{~nm}$ UV irradiation from a hand-held UV lamp at room temperature. Conditions: solution concentration: $10^{-5} \mathrm{M}$; excitation: $440 \mathrm{~nm} . \mathrm{d}$ ) Illustration of the fabrication of ASCPI@ starch microparticles. e-g) Brightfield microscopy image (e), Fluorescence microscopy image (f), SEM image (g) of the ASCPI@ starch microparticles. Scale bar: $50 \mu \mathrm{m}$.

The starch granules usually have spherical, ellipsoidal, or polygonal shapes, ${ }^{40-41}$ which can function as as whispering gallery mode (WGM) resonators providing optical feedback for laser oscillations $^{21,42-45}$. In this study, we explored the possibility of fabricating AIEgen-based microlaser using the starch granules as the WGM resonators. The synthetic procedure of ASCPI@ starch microparticles is illustrated in Figure 1d. Starch granules were sonicated in water. ASCPI was then added into the starch/ $\mathrm{H}_{2} \mathrm{O}$ solution, shaken by hand and left at ambient temperature for $24 \mathrm{~h}$. The obtained ASCPI@ starch particles was centrifuged and washed with pure water. The doping concentration of ASCPI was $1.1 \mathrm{wt} \%$ in starch microparticles. As shown in the bright-field image (Figure 1e), micro-sized ASCPI@starch particles with the ellipsoidal shape could be obtained. ASCPI was weakly emissive in water (Figure S2, Supporting Information). This is possibly attribute to both its AIE activity and ICT property. Since this probe carries a positive charge, it is loosely packed in water. The relatively active intramolecular motion weakens its emission. Moreover, due to its ICT property, its emission is further quenched by water. Therefore, its emission in water is rather weak. When interacting with starch granules, the dye binds to the starch and the strong host-guest interaction between starch and dyes not only restricts 
the intramolecular motions of the AIEgen but also changes its microenvironment. Therefore, the fluorescence of ASCPI in the starch granules was turned on (Figure S2, Supporting Information). As shown in Figure 1f, bright orange-red fluorescence of the particles could be observed under UV excitation, revealing that the ASCPI molecules were successfully encapsulated by starch granules. The scanning electron microscopy (SEM) image (Figure 1g) shows that these ASCPI@starch microparticles possessed perfect ellipsoidal shapes and ultra-smooth surfaces, possibly triggering WGM lasing at a low threshold.
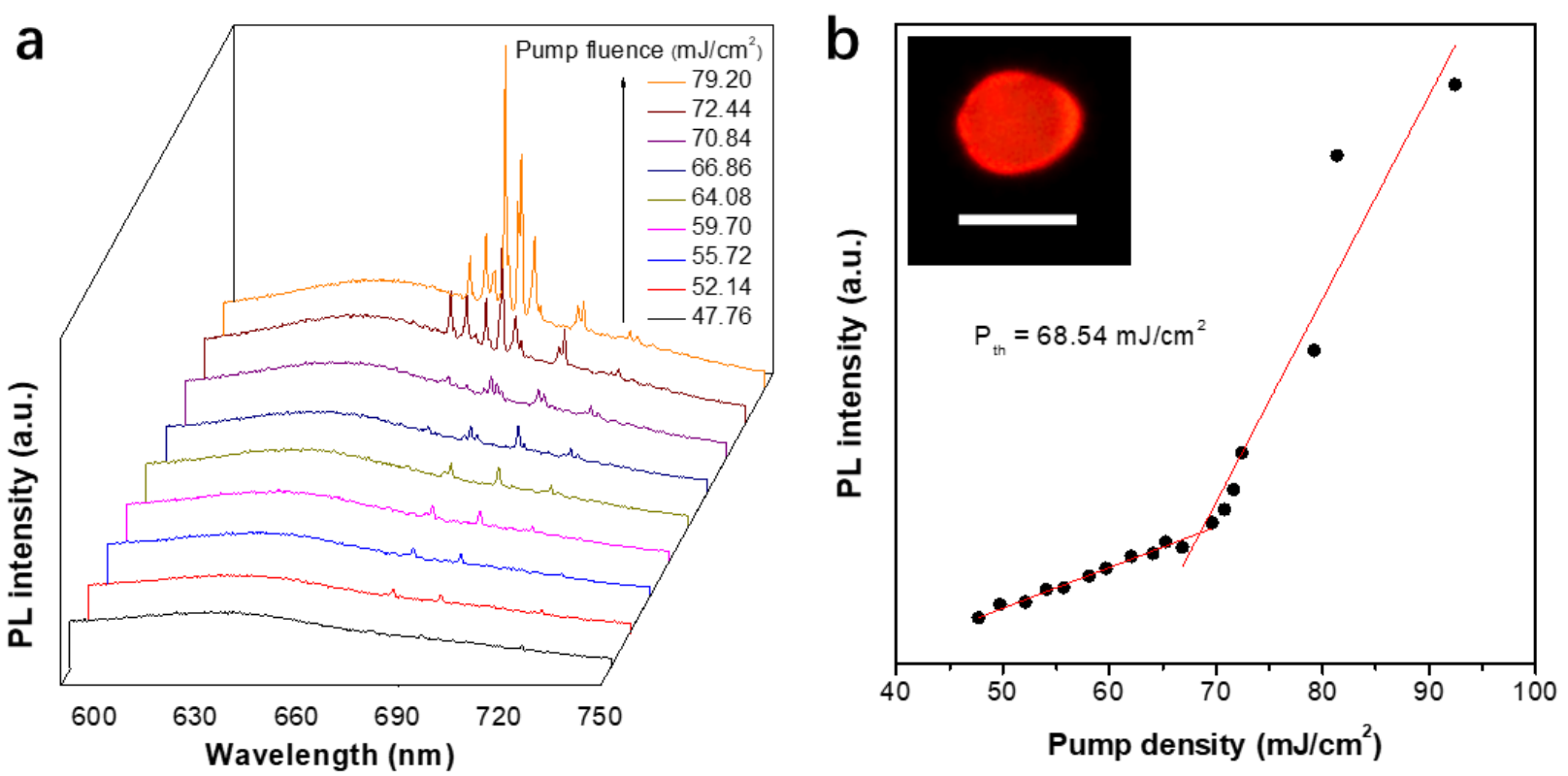

Figure 2. a) PL spectra recorded at the edge of an ASCPI@starch microparticle as a function of pump density. b) Plot of PL peak intensity as a function of pump density. Inset: PL image of the ASCPI@starch microparticle (major axis $10.7 \mu \mathrm{m} \times$ minor axis $8.89 \mu \mathrm{m}$ ) under $400 \mathrm{~nm}$ pulse laser excitation. Scale bar is $10 \mu \mathrm{m}$. 
We then tested optically pumped laser behaviors of these ASCPI@starch microparticles. Here the ASCPI@starch microparticle with dimensions of $10.7 \mu \mathrm{m}$ (major axis) × $8.89 \mu \mathrm{m}$ (minor axis) was excited by a femtosecond pulsed laser beam $(\lambda=400 \mathrm{~nm}, 200 \mathrm{fs})$ with increasing fluence. At low pump fluence, the ASCPI@starch microparticle exhibits a broad emission with spectral peak centered at about $636 \mathrm{~nm}$, corresponding to spontaneous emission (Figure 2a). At higher pump fluence, the emission spectrum of the ASCPI@starch microparticle as a set of sharp peaks at about $680 \mathrm{~nm}$ accompanied by a dramatical enhanced intensity, which indicates stimulated emission. The corresponding PL peak intensity versus pump fluence (Figure $2 b$ ) reveals a clear threshold behavior at about $68.5 \mathrm{~mJ} / \mathrm{cm}^{2}$, which further confirms the lasing action in the microparticles. Above the lasing threshold, the full width at half-maximum (FWHM) at $682 \mathrm{~nm}$ dramatically narrowed down to $\sim 0.4 \mathrm{~nm}$, revealing a sharp increase of temporal coherence.
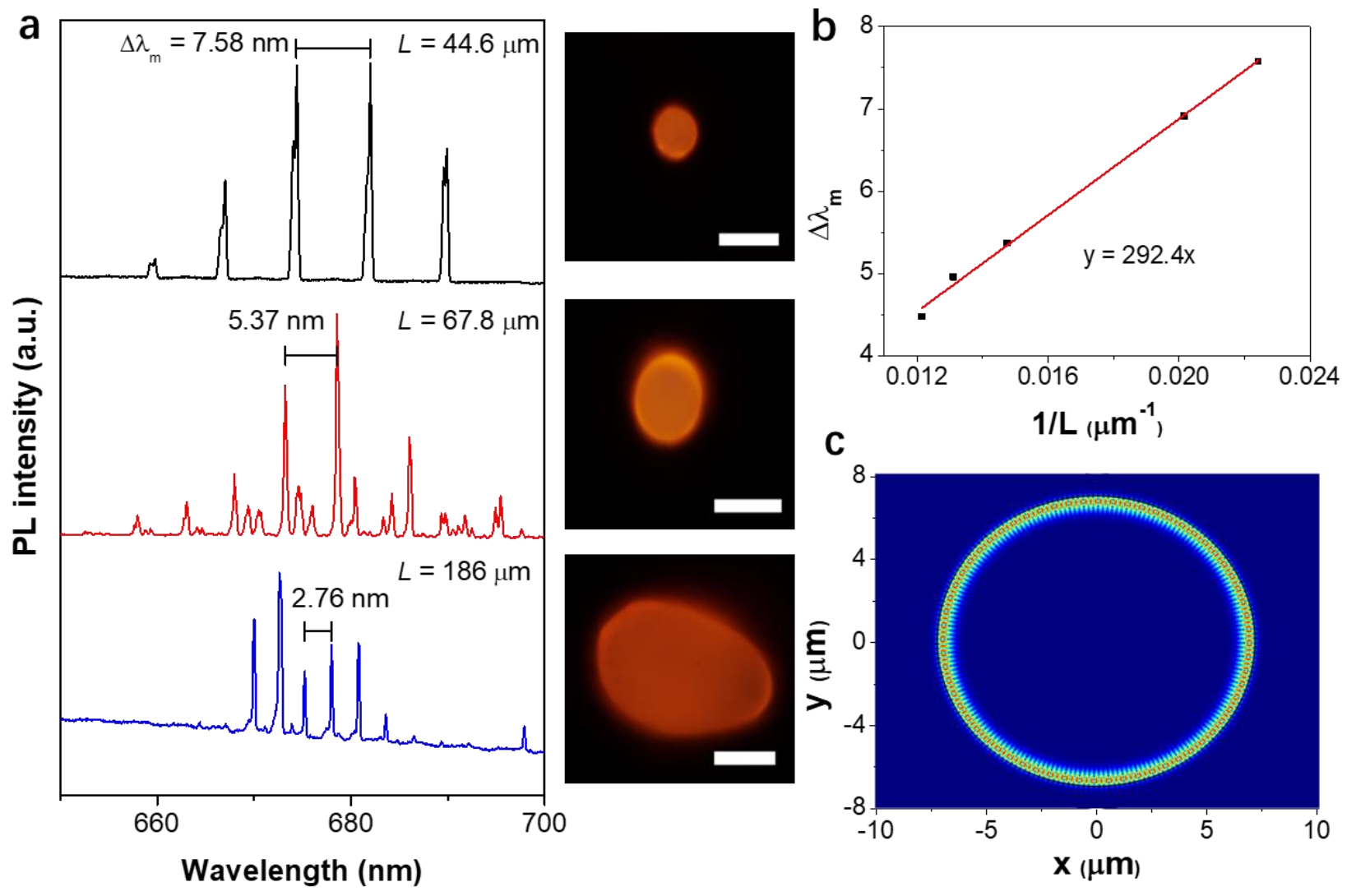
Figure 3. a) PL spectra of the ASCPI@starch microparticles with three different sizes. Corresponding PL images are depicted as insets. All scale bars are $20 \mu \mathrm{m}$. b) Relationship between $\Delta \lambda_{\mathrm{m}}$ and $1 / L$ of the ASCPI@starch microparticles. $L$ is the cavity length. The red line is a fitting to the function $\Delta \lambda_{\mathrm{m}}=\lambda^{2} / n_{\mathrm{g}} L, \lambda=682 \mathrm{~nm}$. c) Simulated field intensity distribution $(\lambda, 682 \mathrm{~nm})$ in an ASCPI@starch microparticle with length of $44.6 \mu \mathrm{m}$.

The lasing spectra of the ASCPI@starch microparticles with different diameters were further studied to investigate the microcavity effects. Figure $3 \mathrm{a}$ illustrates PL spectra and the corresponding images of the three ASCPI@starch microparticles with different dimensions. The mode spacing $\left(\Delta \lambda_{\mathrm{m}}\right)$ between two adjacent lasing modes decreased as the microparticle circumference $(L)$ increased from 44.6 to $186 \mu \mathrm{m}$ (Figure 3a). For WGM resonance, $\Delta \lambda_{\mathrm{m}}$ and $L$ satisfy the equation of $\Delta \lambda_{\mathrm{m}}=\lambda^{2} / n_{\mathrm{g}} L,{ }^{21}$ where $\lambda$ is the wavelength of guided light, $n_{\mathrm{g}}$ is the group refractive index. As shown in Figure $3 b$, the plot $\Delta \lambda_{m}$ and $1 / L$ showed a clear linear relationship. By fitting the plot, the group refractive index $n_{\mathrm{g}}$ is identified with a value of $\sim 1.58$ at $\lambda=682 \mathrm{~nm}$ (Figure 3b), which is consistent with the intrinsic refractive index of the starch polymer $(1.53)^{46}$. These microcavity effect results verified the WGM-type cavity resonance in the ASCPI@starch microparticles. The simulated electric field intensity distribution shown in Figure $3 \mathrm{c}$ further confirms the existence of WGM in the microparticle. 

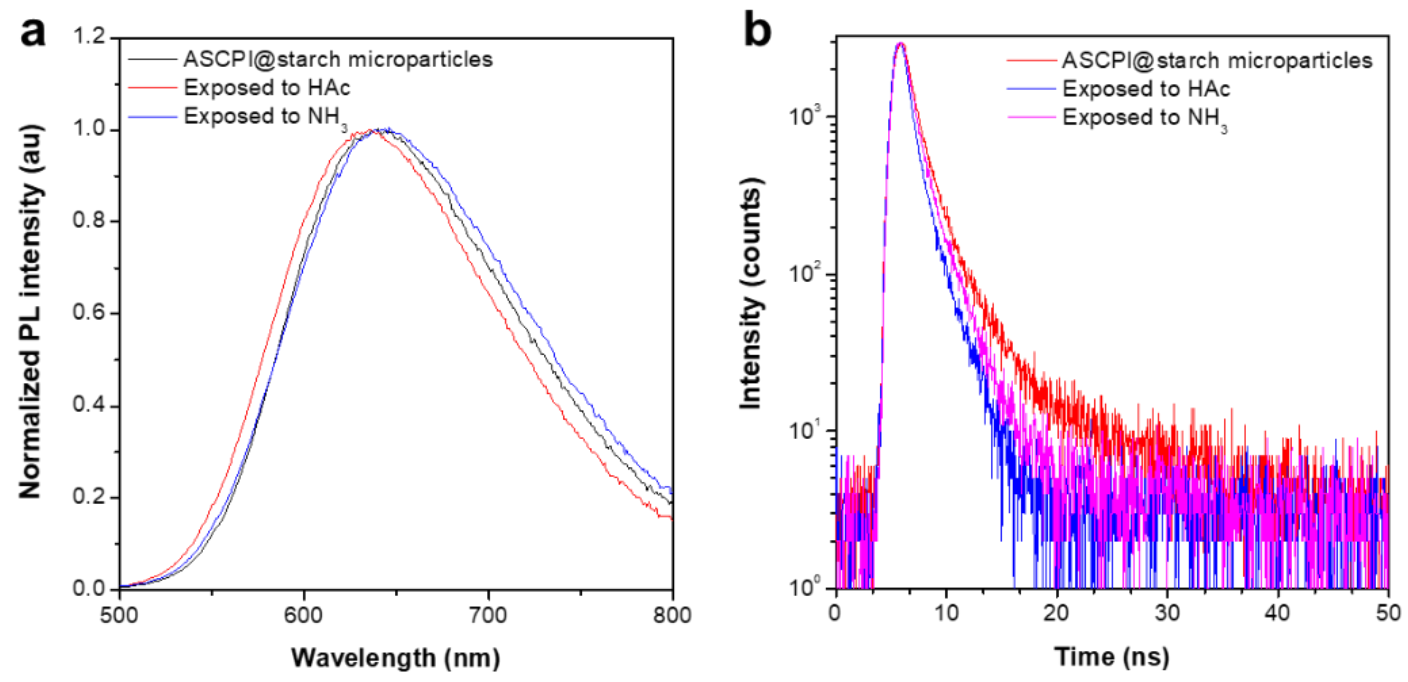

C
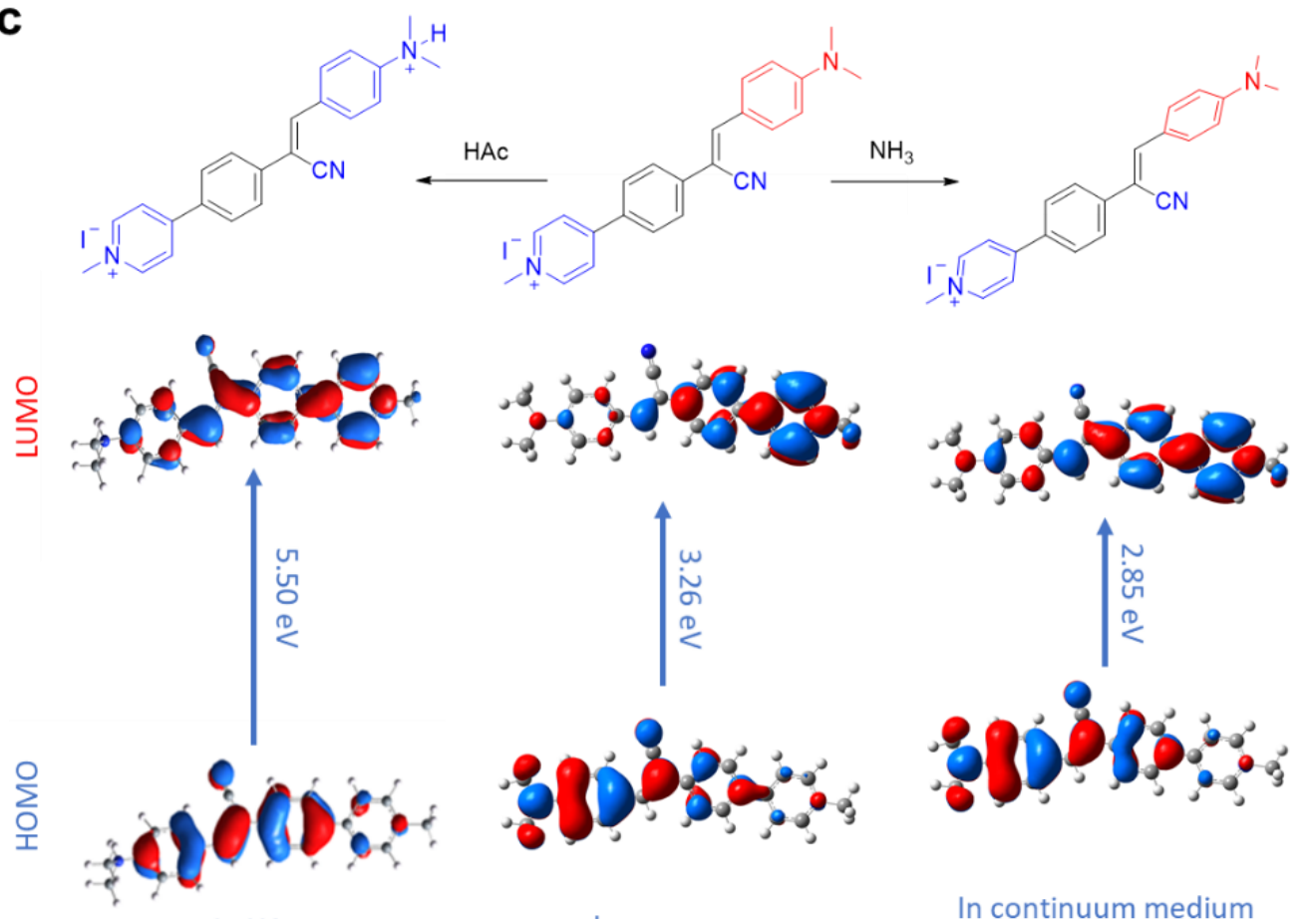

In HAc

In vacuum

In continuum medium with $\varepsilon=17 / \mathrm{n}=1.332$

Figure 4. a) PL spectra of the ASCPI@starch microparticles, ASCPI@starch microparticles in HAc and in $\mathrm{NH}_{3}$ atmosphere. b) PL decay profiles of the ASCPI@ starch microparticles without/with exposure to $\mathrm{HAc}$ and $\mathrm{NH}_{3}$. c) Pictorial presentations of the HOMOs and LUMOs in the ASCPI, ASCPI-HAc complex and ASCPI in $\mathrm{NH}_{3}$ atmosphere. 
As the lasing wavelength is related to the energy gap of optical gain material, it is possible to control the gain region by tuning the energy levels of AIEgens. The ASCPI@ starch microparticle exhibited a broad emission with the wavelength peak at $638 \mathrm{~nm}$ (Figure 4a). After long exposure to HAc, the fluorescence peak of the ASCPI@ starch microparticle exhibited a blue-shift from 638 to $633 \mathrm{~nm}$ along with a decrease of photoluminescence quantum yield (PLQY) from 14.7\% to 10.6\%. In addition, the photoluminescence of the ASCPI@starch microparticle showed bathochromic shift of maximum emission wavelength from 638 to $646 \mathrm{~nm}$ (Figure 4a) and PLQY decreased from $14.7 \%$ to $7.6 \%$ in $\mathrm{NH}_{3}$ atmosphere. This suggests that the external acid/alkaline gas in the atmosphere can tune the energy levels of AIEgens in the ASCPI@starch microparticle. In addition, the fluorescence lifetime of ASCPI@starch microparticle was also investigated to elucidate the inherent excited states property. As shown in Figure 4b, the fluorescence lifetime of ASCPI@starch microparticle was 1.41 ns. Upon exposure to $\mathrm{HAc}$ and $\mathrm{NH}_{3}$, however, the fluorescence lifetime of the ASCPI@starch microparticle decreased to 1.03 and 1.30 ns, respectively. According to the equation $k_{\mathrm{nr}}=(1-\varphi) / \tau$, where $\varphi$ is the PLQY, $k_{\mathrm{nr}}$ is non-radiative decay rate constant, and $\tau$ is fluorescence lifetime, ${ }^{47}$ the non-radiative decay rate constant $\left(k_{\mathrm{nr}}\right)$ of the ASCPI@ starch microparticle increases after exposing to $\mathrm{HAc}$ and $\mathrm{NH}_{3}$ (Table S1). This reveals that the intramolecular rotational motions of AIEgens non-radiatively dissipate the excited-state energy in loose packings of microparticles in $\mathrm{HAc}$ or $\mathrm{NH}_{3}$ atmosphere. To gain more insights into the photophysical mechanism of wavelength switching in $\mathrm{HAc}$ or $\mathrm{NH}_{3}$ atmosphere, the frontier molecular orbitals distributions and energy levels of ASCPI were investigated by density functional theory (DFT) using M062X/def2vp method (Figure 4c) ${ }^{48}$. According to the calculation results, the energy gap of protonated ASCPI was $5.50 \mathrm{eV}$ via combining with protonic acid, which 
was higher than that in vacuum $(3.26 \mathrm{eV})$, showing good agreement with the corresponding optical spectra after the treatment with HAc vapors. However, the energy gap of ASCPI was $2.85 \mathrm{eV}$ in $\mathrm{NH}_{3}$ environment which was lower than that in vacuum $(3.26 \mathrm{eV})$ due to the ICT effect. Therefore, a red-shifted PL was observed in the optical spectra (Figure 4a).
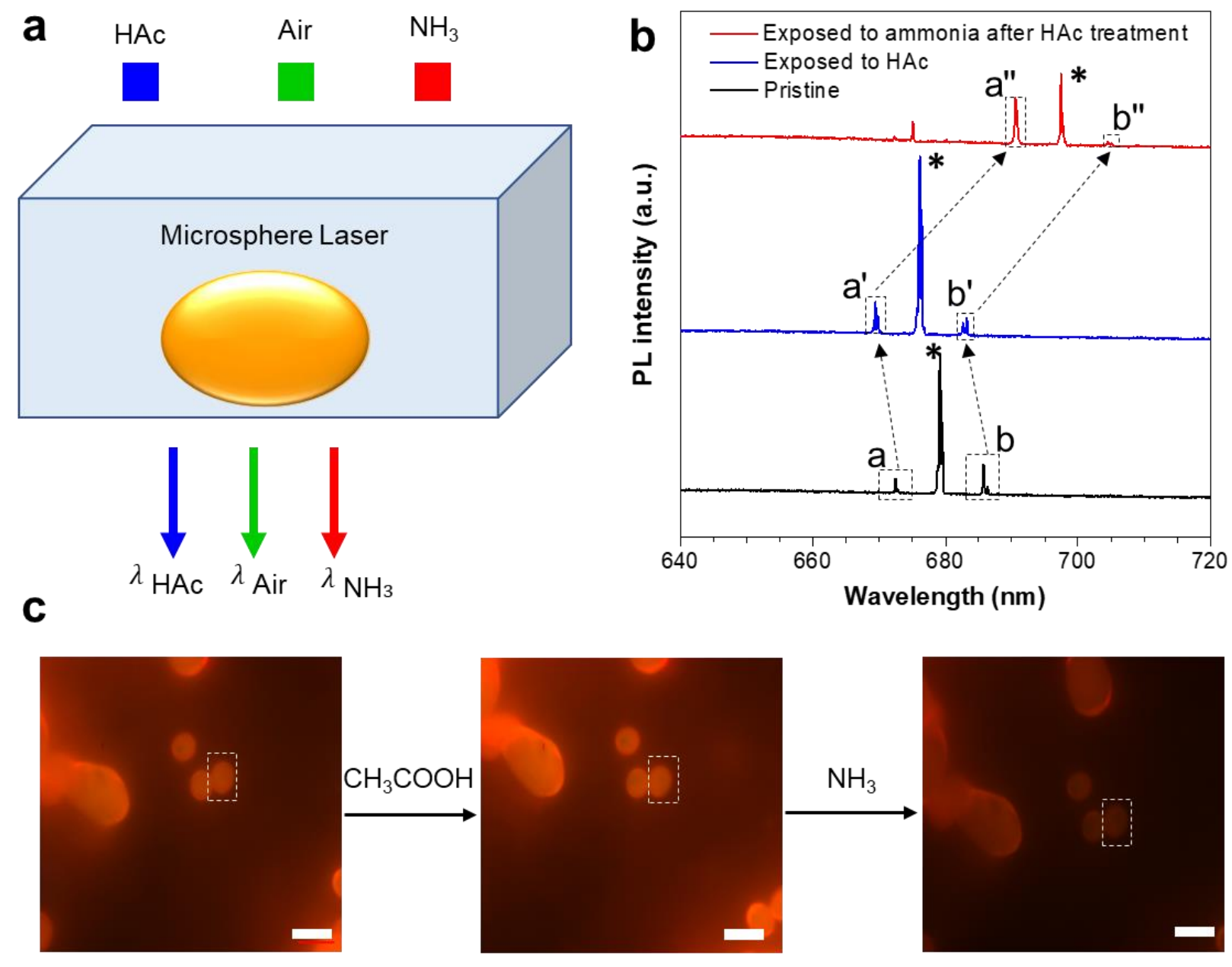

Figure 5. a) Schematic diagram of the ASCPI@starch microsphere laser measurement under exposure to different atmospheres. b) Wavelength shift of lasing modes in the ASCPI@starch microsphere under alternate exposure to $\mathrm{HAc}, \mathrm{NH}_{3}$ and air, respectively. c) PL images of an ASCPI@ starch microsphere under alternate exposure to $\mathrm{HAc}, \mathrm{NH}_{3}$ and air. Scale bars: $20 \mu \mathrm{m}$. 
Inspired by the $\mathrm{HAc} / \mathrm{NH}_{3}$-responsive emission, we measured the lasing spectra in response to the variation of the $\mathrm{HAc}$ or $\mathrm{NH}_{3}$ atmosphere (Figure 5a). Figure $5 \mathrm{~b}$ shows the lasing spectra of a typical ASCPI@starch microsphere (as shown in dotted portion of Figure 5c) with alternating plugs of air, $\mathrm{HAc}$ and $\mathrm{NH}_{3}$ gases. The lasing wavelength of the ASCPI@ starch microsphere was tuned from about 679 to $676 \mathrm{~nm}$ when the microsphere was exposed to HAc gas (Figure 5b). Once the ASCPI@ starch microsphere was exposed to $\mathrm{NH}_{3}$ gas, the lasing wavelength changed to about $691 \mathrm{~nm}$ (Figure 5b). Additionally, as the $\mathrm{HAc}$ or $\mathrm{NH}_{3}$ vapor in the surrounding medium evaporated in air after 10 min, the PL images of the ASCPI@ starch microsphere did not show obvious color change due to the weak fluorescence wavelength switching (Figure 5c). More importantly, compared with the fluorescence wavelength switching, microlaser used as a sensor possessed higher sensitivity to $\mathrm{HAc}$ and $\mathrm{NH}_{3}$ gas stimuli, which could be ascribed to the narrower FWHM in lasing spectra (Figure $4 \mathrm{a}$ and $5 \mathrm{~b}$ ). To further confirm the adaptability of $\mathrm{HAc} / \mathrm{NH}_{3}$-responsive stimulated lasing properties, ASCPI@starch microspheres with different sizes were investigated under external $\mathrm{HAc}$ or $\mathrm{NH}_{3}$ atmosphere (Figure S3-S4). As shown in Figure S3, about $3 \mathrm{~nm}$ of blue-shift was observed after the exposure of the ASCPI@ starch microsphere to HAc gas. On the other hand, other sizes of the ASCPI@ starch microspheres exhibited about $7 \mathrm{~nm}$ bathochromicshift after exposing the microspheres to $\mathrm{NH}_{3}$ gas (Figure S4). Overall, stimulated laser emission responsive to $\mathrm{HAc} / \mathrm{NH}_{3}$ gas can be revealed in the AIEgen@ starch microspheres.

To eliminate the influence of the starch resonator in these switching processes, X-ray diffraction (XRD) patterns of the ASCPI@ starch microsphere treated with acetic acid or ammonia were also investigated, as shown in Figure S5 (Supporting Information). The XRD pattern of the ASCPI@ starch microparticle contained a series of 5.86, 17.2, 22.5 and $24.0^{\circ}$, which was in agreement with that of A-type starches. ${ }^{49}$ It was also found that the diffraction peaks at $2 \theta=17.2$, 
22.5 and $24.0^{\circ}$ of the ASCPI@ starch microparticle had no obvious changes after treating with acetic acid or ammonia, suggesting the structure of A-type starches can be maintained regardless of the influence by $\mathrm{NH}_{3}$ or HAc. In addition, the diffraction peak at $2 \theta=5.86^{\circ}$ disappeared due to the long-range ordered structure of A-type starches was destroyed in the HAc treatment. These data imply that starch used as WGM resonators has little effect on lasing wavelength switching even with the treatment by acid or alkaline.

\section{CONCLUSIONS}

In summary, we reported an AIEgen-based switchable microlaser. This microlaser could be easily fabricated by mixing the AIE active dye ASCPI and starch granules. In the confined environment after binding to the host molecule, starch, the emission of the AIE dye ASCPI was greatly enhanced. We demonstrate that the resultant AIEgen@starch microspheres functioned as typical WGM lasers. More interestingly, manipulation of the laser emission could be achieved by $\mathrm{HAc} / \mathrm{NH}_{3}$ gas treatments. The protonic acid gas could weaken the electron density of the donor system and led to the blue-shifted laser behavior. On the other hand, alkaline gas $\left(\mathrm{NH}_{3}\right)$ provided a more polar environment for these ICT chromophores and thus led to the red-shifted lasing. This work presents a simple method for the fabircation of an efficent AIEgen-based switchable microlaser. It is expected that more AIE-based microlasers and AIE-microlaser-based chemo- or bio-sensors will be developed in the near future.

\section{ASSOCIATED CONTENT}


Supporting Information. The Supporting Information is available free of charge at. UV-Vis, PL spectra, PL image, XRD, Photophysical properties and renal clearance (PDF).

\section{AUTHOR INFORMATION}

\section{Corresponding Authors}

Hui Gao - School of Aeronautic Science and Engineering, Beihang University, Beijing 100191, China; E-mail: h.gao@buaa.edu.cn

Yong Sheng Zhao - Key Laboratory of Photochemistry, Institute of Chemistry, Chinese Academy of Sciences, Beijing 100190, China; E-mail: yszhao@iccas.ac.cn

Sijie Chen - Ming Wai Lau Centre for Reparative Medicine, Karolinska Institutet, Hong Kong, China; E-mail: $\underline{\text { sijie.chen@ki.se }}$

\section{Authors}

Fengyan. Song - School of Aeronautic Science and Engineering, Beihang University, Beijing 100191, China

Chunhuan. Zhang - Key Laboratory of Photochemistry, Institute of Chemistry, Chinese Academy of Sciences, Beijing 100190, China

Haiyun Dong - Key Laboratory of Photochemistry, Institute of Chemistry, Chinese Academy of Sciences, Beijing 100190, China

Yuqin Fan - Key Laboratory of Photochemistry, Institute of Chemistry, Chinese Academy of Sciences, Beijing 100190, China 
Ming-Yu Wu - Ming Wai Lau Centre for Reparative Medicine, Karolinska Institutet, Hong Kong, China

Guogang Shan - Institute of Functional Material Chemistry, Faculty of Chemistry, Northeast Normal University, Renmin Road 5268, Changchun 130024, P.R. China.

Puxiang Lai - Department of Biomedical Engineering, The Hong Kong Polytechnic University, Hong Kong, China.

\section{Author Contributions}

S. Chen. Y. S. Zhao. and H. Gao conceived the idea and designed the study. M-Y. Wu synthesized the compound of ASCPI. F. Song carried out SEM, XRD, UV, PL, PLQY and Lifetime experiments. H. Gao carried out ASCPI@starch microsphere synthesis. C. Zhang, H. Dong and Y. Fan preformed the Lasing measurements. G. Shan and S. Chen carried out DFT calculation. All authors were involved in result discussion and manuscript preparation. F. Song., H. Gao., C. Zhang., S. Chen., and P. Lai. drafted the manuscript with inputs and comments from all authors.

\section{Notes}

The authors declare no competing financial interest.

\section{ACKNOWLEDGMENT}

The authors would like to thank Dr. Chuen Kam for critical reading of the manuscript. C. Zhang acknowledges the financial support from National Natural Science Foundation of China (Grant No. 51903238). H. Gao gratefully acknowledges the financial support from Fundamental Research Funds of Beihang University (Grant No. KG16007908). S. Chen and P. Lai acknowledges the support from the Innovation and Technology Commission (ITS/022/18, ITC, HK). S. Chen 
acknowledges the start-up funding from Ming Wai Lau Centre for Reparative Medicine, Karolinska Institutet.

\section{REFERENCES}

1. Ma, R.-M.; Ota, S.; Li, Y.; Yang, S.; Zhang, X., Explosives detection in a lasing plasmon nanocavity. Nat. Nanotechnol. 2014, 9 (8), 600-604.

2. He, L.; Özdemir, Ş. K.; Zhu, J.; Kim, W.; Yang, L., Detecting single viruses and nanoparticles using whispering gallery microlasers. Nat. Nanotechnol. 2011, 6 (7), 428-432.

3. Humar, M.; Hyun Yun, S., Intracellular microlasers. Nat. Photonics 2015, 9 (9), 572-576.

4. Fan, F.; Turkdogan, S.; Liu, Z.; Shelhammer, D.; Ning, C. Z., A monolithic white laser. Nat. Nanotechnol. 2015, 10 (9), 796-803.

5. $\quad$ Liu, Z.; Yin, L.; Ning, H.; Yang, Z.; Tong, L.; Ning, C.-Z., Dynamical ColorControllable Lasing with Extremely Wide Tuning Range from Red to Green in a Single Alloy Nanowire Using Nanoscale Manipulation. Nano Lett. 2013, 13 (10), 4945-4950.

6. Zhou, Z.; Zhao, J.; Du, Y.; Wang, K.; Liang, J.; Yan, Y.; Zhao, Y. S., Organic Printed Core-Shell Heterostructure Arrays: A Universal Approach to All-Color Laser Display Panels. Angew. Chem. Int. Ed. 2020, 59 (29), 11814-11818.

7. $\quad$ Khalatpour, A.; Paulsen, A. K.; Deimert, C.; Wasilewski, Z. R.; Hu, Q., High-power portable terahertz laser systems. Nat. Photonics 2020.

8. Zhao, L.; Gao, Y.; Su, M.; Shang, Q.; Liu, Z.; Li, Q.; Wei, Q.; Li, M.; Fu, L.; Zhong, Y.; Shi, J.; Chen, J.; Zhao, Y.; Qiu, X.; Liu, X.; Tang, N.; Xing, G.; Wang, X.; Shen, B.; Zhang, Q., Vapor-Phase Incommensurate Heteroepitaxy of Oriented Single-Crystal CsPbBr3 on GaN: Toward Integrated Optoelectronic Applications. ACS Nano 2019, 13 (9), 10085-10094.

9. Mi, Y.; Zhong, Y.; Zhang, Q.; Liu, X., Continuous-Wave Pumped Perovskite Lasers. Adv. Opt. Mater. 2019, 7 (17), 1900544.

10. Yang, X.; Shan, Z.; Luo, Z.; Hu, X.; Liu, H.; Liu, Q.; Zhang, Y.; Zhang, X.; Shoaib, M.; Qu, J.; Yi, X.; Wang, X.; Zhu, X.; Liu, Y.; Liao, L.; Wang, X.; Chen, S.; Pan, A., An Electrically Controlled Wavelength-Tunable Nanoribbon Laser. ACS Nano 2020, 14 (3), 3397-3404.

11. Dong, H.; Zhang, C.; Liu, X.; Yao, J.; Zhao, Y. S., Materials chemistry and engineering in metal halide perovskite lasers. Chem. Soc. Rev. 2020, 49 (3), 951-982.

12. Dong, H.; Zhang, C.; Zhao, Y. S., Controlling the Output of Organic Micro/Nanolasers. Adv. Opt. Mater. 2019, 7 (17), 1900037.

13. Zhao, J.; Yan, Y.; Wei, C.; Zhang, W.; Gao, Z.; Zhao, Y. S., Switchable Single-Mode Perovskite Microlasers Modulated by Responsive Organic Microdisks. Nano Lett. 2018, 18 (2), 1241-1245.

14. Zhang, C.; Zou, C.-L.; Dong, H.; Yan, Y.; Yao, J.; Zhao, Y. S., Dual-color single-mode lasing in axially coupled organic nanowire resonators. Sci. Adv. 2017, 3 (7), e1700225.

15. Lv, Y.; Xiong, Z.; Dong, H.; Wei, C.; Yang, Y.; Ren, A.; Yao, Z.; Li, Y.; Xiang, S.; Zhang, Z.; Zhao, Y. S., Pure Metal-Organic Framework Microlasers with Controlled Cavity Shapes. Nano Lett. 2020, 20 (3), 2020-2025. 
16. Lv, Y.; Xiong, Z.; Yao, Z.; Yang, Y.; Xiang, S.; Zhang, Z.; Zhao, Y. S., StericHindrance-Controlled Laser Switch Based on Pure Metal-Organic Framework Microcrystals. $J$. Am. Chem. Soc. 2019, 141 (51), 19959-19963.

17. Gao, Z.; Zhang, W.; Yan, Y.; Yi, J.; Dong, H.; Wang, K.; Yao, J.; Zhao, Y. S., ProtonControlled Organic Microlaser Switch. ACS Nano 2018, 12 (6), 5734-5740.

18. Dong, H.; Zhang, C.; Lin, X.; Zhou, Z.; Yao, J.; Zhao, Y. S., Dual-Wavelength Switchable Vibronic Lasing in Single-Crystal Organic Microdisks. Nano Lett. 2017, 17 (1), 9196.

19. Dong, H.; Wei, Y.; Zhang, W.; Wei, C.; Zhang, C.; Yao, J.; Zhao, Y. S., Broadband Tunable Microlasers Based on Controlled Intramolecular Charge-Transfer Process in Organic Supramolecular Microcrystals. J. Am. Chem. Soc. 2016, 138 (4), 1118-1121.

20. Zhang, W.; Yan, Y.; Gu, J.; Yao, J.; Zhao, Y. S., Low-Threshold Wavelength-Switchable Organic Nanowire Lasers Based on Excited-State Intramolecular Proton Transfer. Angew. Chem. Int. Ed. 2015, 54 (24), 7125-7129.

21. Wei, Y.; Lin, X.; Wei, C.; Zhang, W.; Yan, Y.; Zhao, Y. S., Starch-Based Biological Microlasers. ACS Nano 2017, 11 (1), 597-602.

22. Görrn, P.; Lehnhardt, M.; Kowalsky, W.; Riedl, T.; Wagner, S., Elastically Tunable SelfOrganized Organic Lasers. Adv. Mater. 2011, 23 (7), 869-872.

23. Karl, M.; Glackin, J. M. E.; Schubert, M.; Kronenberg, N. M.; Turnbull, G. A.; Samuel, I. D. W.; Gather, M. C., Flexible and ultra-lightweight polymer membrane lasers. Nat. Commun. 2018, 9 (1), 1525.

24. Huang, Q.; Xu, H.; Li, M.; Hou, Z.; Lv, C.; Zhan, X.; Li, H.; Xia, H.; Wang, H.; Sun, H., Stretchable PEG-DA Hydrogel-Based Whispering-Gallery-Mode Microlaser with Humidity Responsiveness. J. Lightwave Technol. 2018, 36 (3), 819-824.

25. Zhao, L.; Wang, Y.; Yuan, Y.; Liu, Y.; Liu, S.; Sun, W.; Yang, J.; Li, H., Whispering gallery mode laser based on cholesteric liquid crystal microdroplets as temperature sensor. Opt. Commun. 2017, 402, 181-185.

26. Qiao, C.; Zhang, C.; Zhou, Z.; Dong, H.; Du, Y.; Yao, J.; Zhao, Y. S., A Photoisomerization-Activated Intramolecular Charge-Transfer Process for Broadband-Tunable Single-Mode Microlasers. Angew. Chem. Int. Ed. 2020, 59 (37), 15992-15996.

27. Wei, Y.; Dong, H.; Wei, C.; Zhang, W.; Yan, Y.; Zhao, Y. S., Wavelength-Tunable Microlasers Based on the Encapsulation of Organic Dye in Metal-Organic Frameworks. Adv. Mater. 2016, 28 (34), 7424-7429.

28. Luo, J.; Xie, Z.; Lam, J. W. Y.; Cheng, L.; Chen, H.; Qiu, C.; Kwok, H. S.; Zhan, X.; Liu, Y.; Zhu, D.; Tang, B. Z., Aggregation-induced emission of 1-methyl-1,2,3,4,5pentaphenylsilole. Chem. Commun. 2001, (18), 1740-1741.

29. Mei, J.; Leung, N. L. C.; Kwok, R. T. K.; Lam, J. W. Y.; Tang, B. Z., AggregationInduced Emission: Together We Shine, United We Soar! Chem. Rev. 2015, 115 (21), 1171811940.

30. Zhao, Z.; Zhang, H.; Lam, J. W. Y.; Tang, B. Z., Aggregation-Induced Emission: New Vistas at the Aggregate Level. Angew. Chem. Int. Ed. 2020, 59 (25), 9888-9907.

31. Liu, B.; Tang, B. Z., Aggregation-Induced Emission: More Is Different. Angew. Chem. Int. Ed. 2020, 59 (25), 9788-9789.

32. Zhang, H.; Zhao, Z.; Turley, A. T.; Wang, L.; McGonigal, P. R.; Tu, Y.; Li, Y.; Wang, Z.; Kwok, R. T. K.; Lam, J. W. Y.; Tang, B. Z., Aggregate Science: From Structures to Properties. Adv. Mater. 2020, 32 (36), 2001457. 
33. Liu, W.; Yu, H.; Hu, R.; Xu, T.; Lun, Y.; Gan, J.; Xu, S.; Yang, Z.; Tang, B. Z., Microlasers from AIE-Active BODIPY Derivative. Small 2020, 16 (8), 1907074.

34. Gierschner, J.; Varghese, S.; Park, S. Y., Organic Single Crystal Lasers: A Materials View. Adv. Opt. Mater. 2016, 4 (3), 348-364.

35. Zhao, Z.; Su, H.; Zhang, P.; Cai, Y.; Kwok, R. T. K.; Chen, Y.; He, Z.; Gu, X.; He, X.; Sung, H. H. Y.; Willimas, I. D.; Lam, J. W. Y.; Zhang, Z.; Tang, B. Z., Polyyne bridged AIE luminogens with red emission: design, synthesis, properties and applications. J. Mater. Chem. B 2017, 5 (8), 1650-1657.

36. Liu, S.; Zhou, X.; Zhang, H.; Ou, H.; Lam, J. W. Y.; Liu, Y.; Shi, L.; Ding, D.; Tang, B. Z., Molecular Motion in Aggregates: Manipulating TICT for Boosting Photothermal Theranostics. J. Am. Chem. Soc. 2019, 141 (13), 5359-5368.

37. Song, F.; Xu, Z.; Zhang, Q.; Zhao, Z.; Zhang, H.; Zhao, W.; Qiu, Z.; Qi, C.; Zhang, H.; Sung, H. H. Y.; Williams, I. D.; Lam, J. W. Y.; Zhao, Z.; Qin, A.; Ma, D.; Tang, B. Z., Highly Efficient Circularly Polarized Electroluminescence from Aggregation-Induced Emission Luminogens with Amplified Chirality and Delayed Fluorescence. Adv. Func. Mater. 2018, 28 (17), 1800051.

38. Zhao, Z.; Chen, C.; Wu, W.; Wang, F.; Du, L.; Zhang, X.; Xiong, Y.; He, X.; Cai, Y.; Kwok, R. T. K.; Lam, J. W. Y.; Gao, X.; Sun, P.; Phillips, D. L.; Ding, D.; Tang, B. Z., Highly efficient photothermal nanoagent achieved by harvesting energy via excited-state intramolecular motion within nanoparticles. Nat. Commun. 2019, 10 (1), 768.

39. Yu, C. Y. Y.; Zhang, W.; Kwok, R. T. K.; Leung, C. W. T.; Lam, J. W. Y.; Tang, B. Z., A photostable AIEgen for nucleolus and mitochondria imaging with organelle-specific emission. J. Mater. Chem. B 2016, 4 (15), 2614-2619.

40. Tan, Y.; Xu, K.; Li, L.; Liu, C.; Song, C.; Wang, P., Fabrication of Size-Controlled Starch-Based Nanospheres by Nanoprecipitation. ACS Appl. Mater. Interfaces 2009, 1 (4), 956959.

41. Xu, X.; Visser, R. G. F.; Trindade, L. M., Chapter 4 - Starch Modification by Biotechnology: State of Art and Perspectives. In Starch Polymers, Halley, P. J.; Avérous, L., Eds. Elsevier: Amsterdam, 2014; pp 79-102.

42. Yang Zhao, J.; Chen, L.; Li, J.; Chen, H.-H.; Zhang, X.; Zhou, H., Stretchable organic printed microlaser. IOP Conference Series: Earth and Environmental Science 2020, 461, 012092.

43. Mai, H. H.; Nguyen, T. T.; Giang, K. M.; Do, X. T.; Nguyen, T. T.; Hoang, H. C.; Ta, V. D., Chicken albumen-based whispering gallery mode microlasers. Soft Matter 2020.

44. Liu, X.; Ha, S. T.; Zhang, Q.; de la Mata, M.; Magen, C.; Arbiol, J.; Sum, T. C.; Xiong, Q., Whispering Gallery Mode Lasing from Hexagonal Shaped Layered Lead Iodide Crystals. ACS Nano 2015, 9 (1), 687-695.

45. Ta, V. D.; Chen, R.; Sun, H. D., Self-Assembled Flexible Microlasers. Adv. Mater. 2012, 24 (10), OP60-OP64.

46. Capron, I.; Robert, P.; Colonna, P.; Brogly, M.; Planchot, V., Starch in rubbery and glassy states by FTIR spectroscopy. Carbohydr. Polym. 2007, 68 (2), 249-259.

47. Feng, Q.; Li, Y.; Wang, L.; Li, C.; Wang, J.; Liu, Y.; Li, K.; Hou, H., Multiple-color aggregation-induced emission (AIE) molecules as chemodosimeters for $\mathrm{pH}$ sensing. Chem. Commun. 2016, 52 (15), 3123-3126.

48. Zhao, Y.; Truhlar, D. G., The M06 suite of density functionals for main group thermochemistry, thermochemical kinetics, noncovalent interactions, excited states, and 
transition elements: two new functionals and systematic testing of four M06-class functionals and 12 other functionals. Theor. Chem. Acc. 2008, 120 (1), 215-241.

49. Nishiyama, Y.; Putaux, J.-1.; Montesanti, N.; Hazemann, J.-L.; Rochas, C., B $\rightarrow$ A Allomorphic Transition in Native Starch and Amylose Spherocrystals Monitored by In Situ Synchrotron X-ray Diffraction. Biomacromolecules 2010, 11 (1), 76-87.

BRIEFS: A wavelength-switchable microlaser is achieved in AIEgen@starch microspheres based on $\mathrm{HAc} / \mathrm{NH}_{3}$-responsively stimulated laser emission. The protonic acid (HAc) gas can weaken the electron density of the donor system and lead to a blue-shifted gain behavior, whereas alkaline gas $\left(\mathrm{NH}_{3}\right)$ provides a more polar environment for these intramolecular charge transfer (ICT) chromophores and thus leads to a red-shifted gain behavior.

TOC:

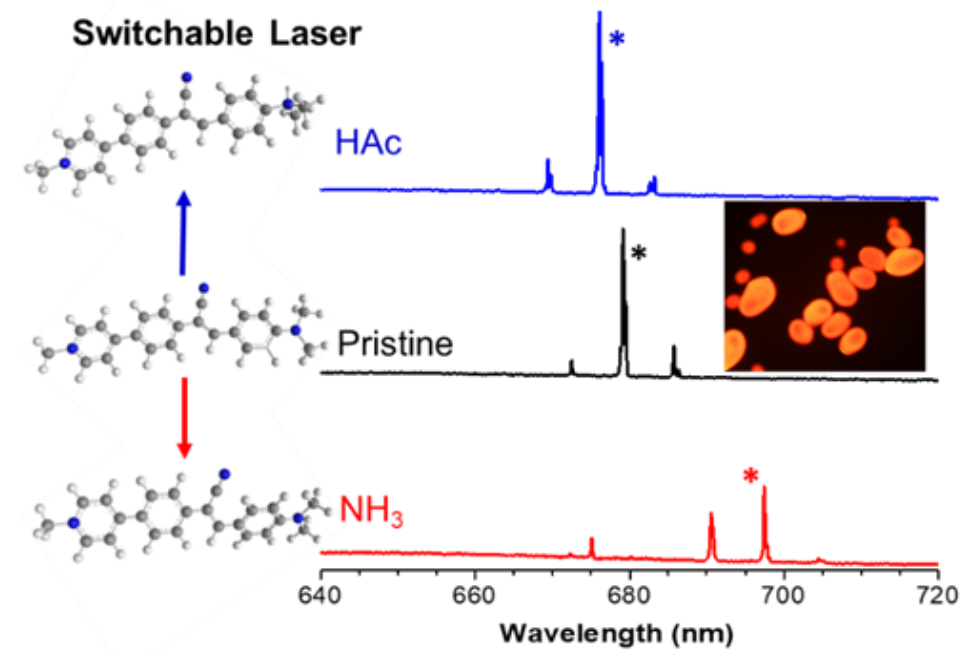

